\title{
Fatores de risco e cuidados à mulher com baby blues
}

\section{Risk factors and attention to women with baby blues}

Roberto NASCIMENTO DE Albuquerque

Daiana VaLéria da Silva ROLLEMBERG ${ }^{\mathrm{I}}$

ICentro Universitário de Brasília

(CEUB), Brasília/DF-Brasil
RESUMO - Introdução: O baby blues é um transtorno mental vivenciado aproximadamente entre 50 e $80 \%$ das mulheres no período pós-parto, com início dos sintomas no primeiro dia do puerpério e regressão de forma espontânea após 10 dias. Essa situação é um importante fator de risco para o desenvolvimento de doenças psicológicas graves, dentre elas a depressão pós-parto (DPP). Objetivo: Verificar os fatores de risco envolvidos no desenvolvimento do baby blues e seus principais cuidados. Material e métodos: Revisão integrativa de literatura realizada entre os meses de dezembro de 2020 e fevereiro de 2021, nas bases de dados Scientific Electronic Library Online (SciELO) e Biblioteca Virtual de Saúde (BVS). Como critérios de inclusão: artigos publicados entre 2011 e 2021, em português, espanhol ou inglês, de acesso gratuito, disponíveis na íntegra e que contemplassem o tema desta pesquisa. Excluíram-se os artigos de revisão, teses ou dissertações. Resultados: Dos 80 artigos encontrados, 48 foram descartados pelo título e por não contemplarem os critérios de inclusão. Após análise criteriosa, 11 foram selecionados para a discussão do presente estudo. A discussão foi dividida em duas partes: O baby blues, seus sinais, sintomas e seus fatores de risco; e os cuidados à mulher com baby blues. Conclusões: Este estudo evidenciou diferentes fatores de risco como questões hormonais, tipos de parto, amamentação, questões psicossociais, fatores relacionais com parceiro/parceira além de dificuldades nos cuidados diretos ao bebê. Além disso, ressalta-se a importância da equipe multidisciplinar para detecção dos fatores de risco, promoção da saúde mental e prevenção do baby blues.

Palavras-chaves: Transtornos Puerperais; Saúde Mental; AsSISTÊNCIA À SAÚde MENTAL.

Abstract - Introduction: The baby blues is a mental disorder experienced by approximately 50 to $80 \%$ of women in the postpartum period, with onset of symptoms on the first day of the puerperium and regresses spontaneously after 10 days. This situation is an important risk factor for the development of serious psychological diseases, including postpartum depression (PPD). Objective: To verify the risk factors involved in the development of baby blues and their main care. Material and Methods: Integrative literature review carried out between the months of December 2020 and February 2021, in the Scientific Electronic Library Online (SciELO) and Virtual Health Library (VHL) 
databases. As inclusion criteria: articles published between 2011 and 2021, in Portuguese, Spanish or English, free access, available in full text and that covered the theme of this research. Review articles, theses or dissertations were excluded. Results: Of the 80 articles found, 48 were discarded by the title and because they did not meet the inclusion criteria. After careful analysis, 11 articles were selected to discuss the present study. The discussion was divided into two parts: The baby blues, its signs, symptoms and its risk factors and; Care for women with baby blues. Conclusions: This study showed different risk factors such as hormonal issues, types of delivery, breastfeeding, psychosocial issues, relational factors with a partner and difficulties in direct baby care. In addition, showed the importance of the multidisciplinary team for detecting risk factors, promoting mental health and preventing baby blues is emphasized.

Keywords: Puerperal Disorders; Mental Health; Mental Health Assistance.

\section{INTRODUÇÃO}

A gestação é uma condição indispensável para sobrevivência da vida humana, pois, através da gravidez, ocorre a renovação das gerações e representa o período de formação de um novo indivíduo. Esse período inicia-se com a fecundação, estende-se por cerca de quarenta semanas e termina com o parto. Ressalta-se que é um momento de profundas mudanças, não apenas na vida pessoal da mulher, mas também na história de toda a família. ${ }^{1}$

A mulher, durante o período gravídico, vivencia diferentes sentimentos e emoções que, muitas vezes, podem ser ocasionados por forte sofrimento psíquico., ${ }^{2,3}$ Nas primeiras semanas de gestação, a mulher pode ter sentimentos intensos e contraditórios como dúvidas sobre estar grávida ou não, tristeza, alegria e/ou enjeitamento ao feto. Já no segundo trimestre, a gestante pode apresentar um estado emocional mais estável, muitas vezes pela certeza da gravidez e o aparecimento dos movimentos do feto. $\mathrm{E}$ por fim, no terceiro trimestre da gravidez, a ansiedade pode aumentar devido à proximidade do parto. ${ }^{4}$
As mulheres que passaram pela primeira vez pela experiência do parto (primíparas) podem apresentar um aumento da ansiedade devido à inexperiência com o processo gestacional; aliados a essa situação, gravidezes não planejadas ou situações socioeconômicas desfavoráveis da mãe podem desenvolver crises de ansiedade nesse período. ${ }^{5}$

Outro momento especial para as mulheres é o puerpério, período evidenciado logo após o parto. Esse período, aproximadamente de 6 a 8 semanas, pode ser dividido em três momentos: o imediato, que consiste no $1^{\circ}$ ao $10^{\circ}$ dia após o parto; o tardio, que ocorre entre o $11^{\circ}$ e o $45^{\circ}$ dia pós-parto e; o remoto, momento a partir do $45^{\circ}$ dia pós-parto. ${ }^{6}$

Durante o puerpério, a mulher pode vivenciar intenso sofrimento psíquico devido a questões como mudanças na fisionomia, necessidades de adaptações pessoais e laborais, bem como exigências específicas do cuidado com o bebê. ${ }^{7}$ Nesses casos, alguns transtornos mentais podem ser evidenciados, tais como o baby blues, a depressão pós-parto e a psicose puerperal. ${ }^{8}$

O baby blues, também conhecido por disforia puerperal ou tristeza materna, foi 
descrito pela primeira vez em 1960. Essa condição foi observada após o parto de uma parcela significativa de mulheres as quais apresentavam choro com facilidade, porém não havia relação direta com sentimento de tristeza. ${ }^{9}$ Os sintomas do baby blues têm início no primeiro dia do puerpério, ocorrendo com maior intensidade por volta do quinto dia, desaparecendo, em média, até do décimo dia. Observa-se que entre $50 \%$ e $80 \%$ das mulheres podem apresentar baby blues durante o puerpério, além disso é um importante fator de risco para depressão no período pós-parto e psicoses puerperais. ${ }^{10,11}$

Essa situação diferencia-se da depressão pós-parto (DPP), pois a DPP é caracterizada como episódios depressivos que ocorrem nos meses após o nascimento da criança, tendo início entre duas semanas até três meses após o parto; os sintomas são a perda de prazer e interesse nas atividades, o sentimento de inutilidade ou culpa, a dificuldade para concentrar-se e presença de pensamentos de morte ou suicídio. ${ }^{9}$ Já a psicose puerperal é um transtorno grave e de prevalência de $0,1 \%$ a $0,2 \%$ das puérperas, tem início rápido (aparece nos primeiros dias até duas semanas após o parto) e os sintomas mais comuns são delírios, ideias persecutórias e alucinações. ${ }^{12}$

Observa-se que as questões emocionais envolvidas durante a gravidez e gestação devem ser bastante observadas e avaliadas pelos profissionais de saúde envolvidos nesse momento tão especial para a mulher. Isso porque o tipo de relação entre a mãe e o seu filho será determinante para a formação de vínculos entre profissionais-mulher e entre o binômio mãe-filho. A existência de sofrimento psíquico ou transtornos mentais por parte da mãe podem influenciar negativamente a saúde mental mater- na, além de intensificar sintomas depressivos e ansiosos. ${ }^{13}$

Como visto anteriormente, um percentual elevado de mulheres pode desenvolver o baby blues durante o puerpério. Dessa maneira, justifica-se este estudo, pois os profissionais de saúde precisam conhecer melhor esse transtorno e criar estratégias de cuidados frente a essa situação.

Frente ao exposto, o presente trabalho teve como objetivo verificar os fatores de risco envolvidos no desenvolvimento do baby blues e os principais cuidados para amenizar tal situação.

\section{MATERIAL E MÉtodos}

Trata-se de uma revisão integrativa de literatura que propõe uma reflexão sobre o baby blues e os fatores que podem influenciar a mulher a desenvolver tal transtorno. Para o seu desenvolvimento, fez-se uso dos pressupostos da revisão integrativa de literatura e teve como questão norteadora: "Quais os fatores de risco que podem levar a mulher a desenvolver o baby blues e o que os profissionais de saúde podem fazer para amenizar tal situação?".

As revisões integrativas de literatura incluem a análise e a síntese de pesquisas de maneira sistematizada de modo a contribuir para o aprofundamento do tema investigado, auxiliar na tomada de decisão e consequentemente, na melhoria da prática clínica, com base em resultados de pesquisa. ${ }^{14}$

Os critérios de inclusão foram: artigos publicados nos últimos dez anos (2011 a 2021), em português, espanhol ou inglês, gratuitos, disponíveis em sua íntegra e que contemplassem o tema proposto para esta pesquisa. Excluíram-se os artigos de revisão, teses ou dissertações. 
A coleta de dados ocorreu entre os meses de dezembro de 2020 e fevereiro de 2021, junto às bases de dados da Scientific Eletronic Library Online (SCIELO) e Biblioteca Virtual de Saúde (BVS). Foram utilizadas as seguintes palavras: transtornos puerperais, depressão pós-parto e baby blues, por meio dos conectores booleanos OR e AND a seguir: "puerperal disorders OR postpartum depression AND baby blues".

Ressalta-se que, apesar da palavra baby blues não ser descritor do DeCs (Descritores de Ciências da Saúde) e do MeSH (Medical Subject Headings), a mesma foi necessária para uma melhor delimitação e seleção dos artigos.

Para alcançar o objetivo proposto, a pesquisa foi dividida em 6 partes: (1) Identificação do tema e definição da questão norteadora; (2) Busca de evidências/artigos conforme critérios de inclusão, exclusão e questão norteadora; (3) Avaliação das evidências dos estudos selecionados; (4) Integração das evidências por meio da construção de um quadro com os artigos selecionados para a revisão integrativa; (5) Discussão dos resultados e; (6) Apresentação da síntese dos conhecimentos.

\section{RESULTADOS}

Foram encontrados 80 artigos nas bases de dados selecionadas. Na BVS, dos 79 artigos, 48 foram descartados pelo título e por não contemplarem os critérios de inclusão da pesquisa. Logo em seguida, foram lidos 31 resumos; 20 deles foram selecionados para leitura na íntegra, e, destes, 10 selecionados para revisão integrativa. Na SCIELO foi encontrado apenas 1 artigo, e o mesmo foi selecionado para esta revisão. Esses dados são encontrados na Figura 1.

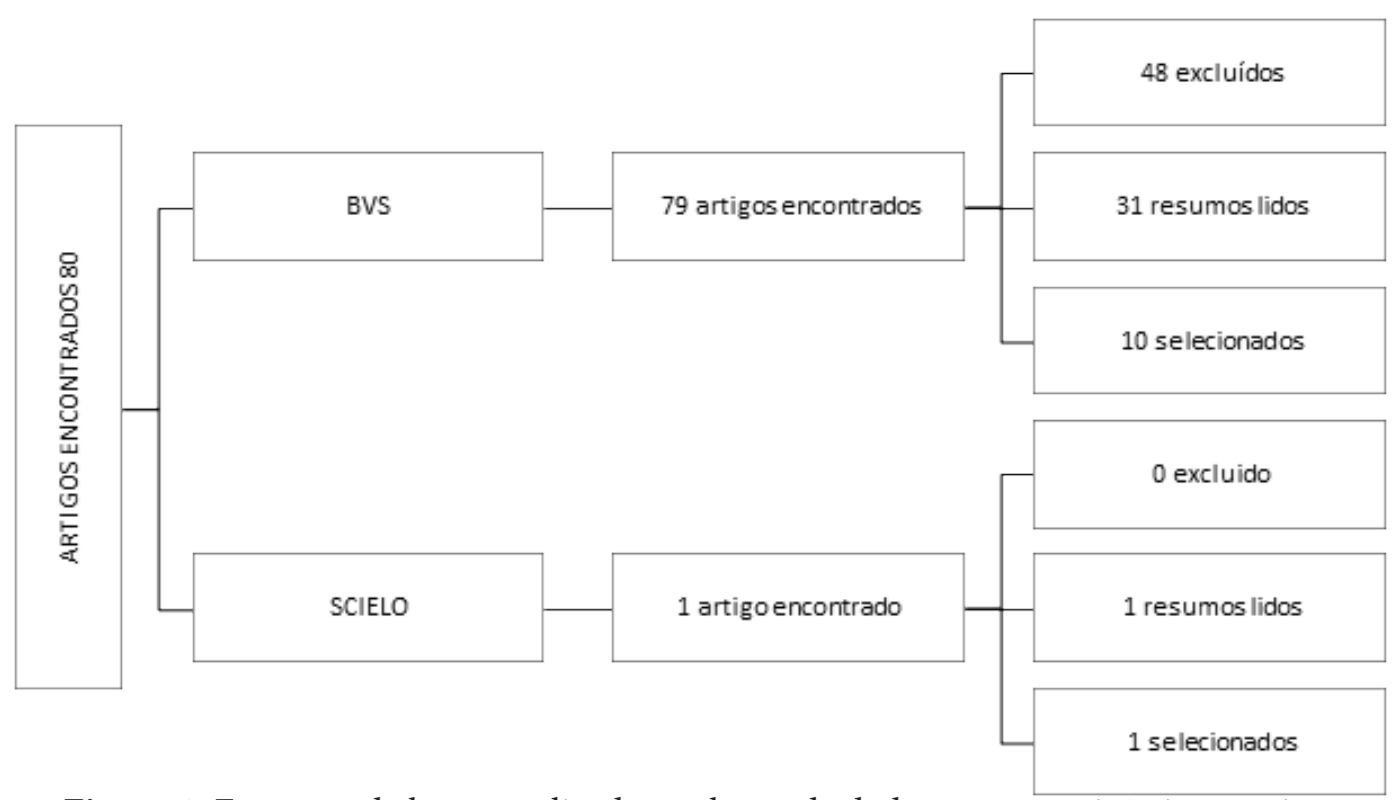

Figura 1: Esquema da busca realizada nas bases de dados para a revisão integrativa.

Dessa maneira, o corpus desta pesquisa foi composto por 11 artigos e que servirão como base para a discussão desta revisão integrativa de literatura, conforme Quadro 1. 
Quadro 1. Síntese dos artigos sobre os fatores de risco e cuidados referentes ao baby blues.

\begin{tabular}{|c|c|c|c|}
\hline $\begin{array}{l}\text { Referência/Ano/País de } \\
\text { Origem }\end{array}$ & Objetivos & Tipo de Estudo & Duração \\
\hline $\begin{array}{l}\text { Zareba et al., } 2020^{15} \text {. } \\
\text { Suíça }\end{array}$ & $\begin{array}{l}\text { Identificar os fatores de risco presentes } \\
\text { durante o parto que possam estar } \\
\text { relacionados aos transtornos de humor } \\
\text { no puerpério }\end{array}$ & Estudo retrospectivo & 7 anos \\
\hline $\begin{array}{l}\text { Pan et al., } 2019^{16} \text {. } \\
\text { Estados Unidos }\end{array}$ & $\begin{array}{l}\text { Avaliar a saúde psicológica das mulheres } \\
\text { durante a gravidez e no pós-parto }\end{array}$ & Ensaio Randomizado & 1 ano \\
\hline $\begin{array}{l}\text { Akbarzedeh et al., } 2018^{17} . \\
\text { Egito }\end{array}$ & $\begin{array}{l}\text { Examinar a influência da amamentação } \\
\text { baseado no modelo BASNEF (modelo } \\
\text { de avaliação de crenças, atitudes, normas } \\
\text { subjetivas e fatores facilitadores) para a } \\
\text { gravidade do baby blues }\end{array}$ & $\begin{array}{l}\text { Estudo Semi- } \\
\text { Experimental }\end{array}$ & 1 ano \\
\hline $\begin{array}{l}\text { Riazanova et al., } 2018^{18} . \\
\text { Romênia }\end{array}$ & $\begin{array}{l}\text { Verificar a correlação ao estresse durante } \\
\text { o parto natural com o aumento do risco } \\
\text { de baby blues }\end{array}$ & Ensaio Randomizado & 2 anos \\
\hline $\begin{array}{c}\text { Dowlati et al., } 2017^{19} \text {. } \\
\text { Estados Unidos } \\
\end{array}$ & $\begin{array}{l}\text { Avaliar se a suplementação alimentar } \\
\text { reduz a vulnerabilidade ao baby blues }\end{array}$ & Ensaio Randomizado & 1 ano \\
\hline $\begin{array}{l}\text { Fiala et al., } 2017^{20} . \\
\text { Estados Unidos }\end{array}$ & $\begin{array}{l}\text { Examinar sinais depressivos e identificar } \\
\text { fatores de risco. }\end{array}$ & Estudo Longitudinal & 10 anos \\
\hline $\begin{array}{l}\text { Silva et al., } 2017^{11} \text {. } \\
\text { Portugal }\end{array}$ & $\begin{array}{l}\text { Analisar a presença de sintomas de } \\
\text { tristeza materna e seus fatores associados }\end{array}$ & Estudo Transversal & 1 ano \\
\hline $\begin{array}{l}\text { Mughal et al., } 2016^{21} . \\
\text { Estados Unidos }\end{array}$ & $\begin{array}{l}\text { Identificar alterações psicológicas no } \\
\text { pós-parto e atuação do enfermeiro nesse } \\
\text { contexto. }\end{array}$ & Ensaio Randomizado & 1 ano \\
\hline $\begin{array}{c}\text { Maliszewska et al.,2016 }{ }^{22} . \\
\text { Polônia }\end{array}$ & $\begin{array}{l}\text { Identificação dos fatores que aumentam } \\
\text { ou diminuem o risco do baby blues }\end{array}$ & Estudo Transversal & 3 meses \\
\hline $\begin{array}{l}\text { Kumar et al., } 2016^{23} . \\
\text { Índia }\end{array}$ & $\begin{array}{l}\text { Avaliar a proporção e os tipos de } \\
\text { morbidade psiquiátricas no pós-parto em } \\
\text { um hospital de atenção terciária }\end{array}$ & Estudo Transversal & 1 ano \\
\hline $\begin{array}{c}\text { Masmoudi et al., } 2014^{24} \text {. } \\
\text { Tunísia }\end{array}$ & $\begin{array}{l}\text { Estimar a prevalência e fatores associados } \\
\text { a depressão pós-parto }\end{array}$ & Estudo Prospectivo & 1 ano \\
\hline
\end{tabular}

\section{Discussão}

\section{O baby blues, seus sinais e sinto- mas e seus fatores de risco}

Observou-se que o baby blues acomete cerca de $80 \%$ das parturientes e pode ser observado no primeiro dia do puerpério por meio da detecção precoce dos fatores de risco. ${ }^{24,23} \mathrm{Um}$ dos primeiros fatores de risco é a possibilidade de uma disfunção do eixo-hipotalâmico-pituitário-adrenal e das mudanças hormonais de estrogênio e progesterona que ocorrem no período gestacional e puerperal. Essa desregulação hormonal pode ocorrer devido à liberação de cortisol 
em casos de trauma e estresse antes, durante e após o parto. ${ }^{21}$

Esses fatores de risco podem estar correlacionados com os sintomas do baby blues, tais como, choro, insônia, tristeza, falta de apetite, fadiga, mudança de peso, perda da libido, baixa concentração e neuroticismo, instabilidade emocional, sentimentos e emoções negativas e baixa adaptação ao estresse. ${ }^{11,17,19,20,21,22,24}$

O parto pode ser esse fator de trauma e estresse. Mulheres que foram submetidas a cesarianas, além daquelas que passaram por partos com utilização de fórceps, espátulas ou ventosas foram mais suscetíveis a desenvolver baby blues do que mulheres que passaram por parto natural. ${ }^{15}$ Além disso, apesar da analgesia peridural auxiliar na diminuição da dor durante o parto, pode estar diretamente correlacionada à liberação de cortisol, no desenvolvimento de sintomatologia depressiva e do aparecimento do baby blues. ${ }^{18}$

Outro fator de risco para o baby blues está relacionado à internação da mulher para o parto. Mulheres que foram hospitalizadas para o período do parto, ou aquelas em que o recém-nascido obtiveram uma baixa pontuação na Escala de APGAR (teste realizado no recém-nascido que avalia seu estado geral e vitalidade) no pós-parto imediato, apresentaram uma maior suscetibilidade de transtornos psicológicos durante o puerpério, dentre eles o baby blues. Tal fato se explica pela ansiedade materna vivenciada durante a hospitalização e o sentimento de impotência de reverter tal situação do recém-nascido. ${ }^{15}$

Gravidezes de alto risco e partos prematuros também podem aumentar o sofrimen- to psíquico da mulher. Estudos demonstraram que a perda sanguínea acima de $400 \mathrm{ml}$ durante o parto aumentou a ansiedade, o medo e o estresse da mulher. Essa situação foi um fator importante para o desenvolvimento da tristeza materna. ${ }^{15,21}$

A impossibilidade de realizarem a amamentação também foi um fator de risco relevante para o aparecimento do baby blues. Mulheres que não amamentaram foram mais suscetíveis a apresentarem alterações psicológicas durante o período puerperal, pois, durante a amamentação, ocorre a liberação de ocitocina e prolactina. Tais hormônios diminuem os sintomas depressivos, de estresse e de ansiedade na mulher. ${ }^{17,20}$

Além disso, fatores psicossociais e econômicos também foram vistos como fatores de risco o baby blues. Gravidez não planejada, baixa escolaridade da mãe, ausência de companheiro/companheira (mãe solteira), divergência de opiniões entre parceiros/ parceiras e familiares, desemprego ou renda abaixo de R\$ 150,00 mensais, feto do sexo feminino, histórico pessoal ou familiar de transtornos mentais prévios (sendo eles puerperais ou não), baixa autoestima, primíparas e dificuldades relacionadas aos cuidados direitos com o recém-nascido também foram importantes fatores de risco para o desenvolvimento de transtornos puerperais, dentre eles o baby blues. ${ }^{11,19,20,22}$

\section{Cuidados à mulher com baby blues}

Medidas psicoeducativas e psicoterapêuticas com a mulher são necessárias nesse momento tão especial. Essas ações devem ser incluídas desde as consultas de pré-natal até os primeiros meses de vida da criança. ${ }^{15,24}$ 
As consultas de pré-natal têm um papel fundamental para a diminuição dos casos de baby blues. Durante essas consultas, a gestante deve ser incentivada a partilhar seus medos, anseios e angústias com os profissionais de saúde. Essa ação melhora a criação de vínculos, além de auxiliar a mulher nesse novo período que é a maternagem. ${ }^{22}$

Uma estratégia eficaz para a redução do estresse psicológico é a implantação de programas baseados na atenção plena à saúde mental chamados Mindfulness, técnicas de meditação que colaboram para o autocontrole emocional. Esse programa já é implementado nos países desenvolvidos, e ensina mulheres e seus/suas companheiros/companheiras a monitorarem seus estados emocionais, cognitivos e de autoconsciência para o processo da maternidade/paternidade. ${ }^{16}$

Outro cuidado auxiliar na diminuição da tristeza materna é a suplementação dietética e a prática de exercícios físicos. A inclusão de mirtilo, tirosina e triptofano na dieta auxiliaram na liberação de serotonina, norepinefrina e dopamina; além disso diminuíram a ação da Monoamina Oxidase-A, isoenzima capaz de clivar os neurotransmissores que influenciam o humor. ${ }^{19}$ Além disso, a criação de kits alimentares de baixo custo pode ajudar as mulheres na melhora do humor e consequentemente na prevenção do baby blues, o estudo ainda sugere que a prática de exercício físico aliado ao apoio psicossocial poderia ser fornecido simultaneamente com a suplementação para redução do estresse. ${ }^{19}$

Orientações sobre os benefícios da amamentação também são cuidados importantes para a redução dos casos de baby blues. Cursos de aleitamento materno auxiliam redução de dúvidas relacionadas à técnica correta da amamentação, da ordenha e armazenamento do leite materno, da presença de dor na amamentação, da quantidade de leite materno adequado para o recém-nascido, da importância da criação de vínculo entre mãe e filho, além de explicar sobre os hormônios ocitocina e prolactina liberados durante a amamentação e seus efeitos antidepressivos e ansiolíticos para a mulher. ${ }^{15,17}$

Ações que promovam o apoio social e profissional também são cuidados importantes pós-parto. A equipe de saúde deve criar estratégias de acolhimento, além de incentivar as mulheres que apresentem sinais e sintomas de baby blues a expressarem seus sentimentos e anseios. Ressalta-se que o aparecimento desse transtorno pode ser um gatilho para o desenvolvimento da depressão pós-parto e demais transtornos puerperais. ${ }^{22}$

Caso a equipe de saúde perceba que o quadro de tristeza tem se agravado, sugere-se a implantação/implementação de diferentes tipos de escalas para avaliação - dentre elas a Escala de Depressão de Edimburgo e de Humor Brasileira; são escalas eficazes para rastreamento e identificação de sintomas depressivos. Com isso, caso seja necessário, encaminhar para os demais profissionais da equipe de saúde: psiquiatras, psicólogos ou enfermeiros especialistas em saúde mental. ${ }^{11}$

\section{CONSIDERAÇõeS FINAIS}

Por meio desse estudo foi possível verificar diferentes fatores de risco para o aparecimento do baby blues tais como: questões hormonais envolvidas na gestação, o momento do parto, tipos de parto, a ama- 
mentação, bem como questões psicossociais como dificuldades econômicas, fatores relacionais com o/a parceiro/parceira, medo do futuro, dificuldades de cuidados diretos com o bebê, dentre outros.

Ficou evidente que além das consultas de pré-natal, a equipe multiprofissional de saúde deve identificar dificuldades financeiras, sociais e psicológicas durante o período pós-parto para prevenir eventuais declínios psíquicos da mulher e, consequentemente, na prevenção do baby blues. Dentre as medidas preventivas a Escala de Edinburgh e a Escala de Humor Brasileira podem ser usadas pelos profissionais, a fim de detectar precocemente alterações psicossociais vivenciadas pelas mulheres no período gestacional e puerperal.

Foi observado que, pesquisas no âmbito da Enfermagem na Saúde da Mulher e Enfermagem em Saúde Mental, o baby blues ainda tem sido pouco explorado, especialmente em estudos na língua portuguesa. Enfermeiros da atenção primária e obstetras devem estar capacitados para identificar precocemente os fatores de risco e os sintomas relacionados ao baby blues, diferenciá-lo de outros transtornos psicológicos relacionados à maternidade e prestar os cuidados necessários para amenizar esse sofrimento psíquico que acomete tantas mulheres.

Portanto, faz-se necessárias novas pesquisas no âmbito da Saúde da Mulher e da Saúde Mental, a fim de melhorar as discussões sobre o baby blues e possíveis situações que podem agravar este quadro, tais como: feto que apresente alguma deformidade congênita ou com síndrome de Down, gravidez na adolescência, duração do trabalho de parto e baby blues prévio.

É necessário a realização de treinamentos e cursos de curta duração que capacite os profissionais de saúde a reconhecer os sintomas e os fatores de riscos já mencionados, pois dessa forma é possível minimizar os efeitos do baby blues nesse período de grandes transformações na vida da mulher.

\section{REFERÊNCIAS}

1 Coutinho EC, Silva CB, Chaves CMB, Nelas PAB, Parreira VBC, Amaral MO et al. Gravidez e parto: O que muda no estilo de vida das mulheres que se tornam mães? Rev Esc Enferm USP [Internet]. 2014 [acesso em 2021 Mar 13]; 48(2):17-24, 2014. Disponível em: https://www.scielo. br/pdf/reeusp/v48nspe2/pt_0080-6234-reeusp-48-nspe2-00017.pdf

2 Camacho KG, Vargens OMC, Progianti JM, Spíndola T. Vivenciando Repercussões E Transformações De Uma Gestação: Perspectivas De Gestantes. Cienc. enferm. xvi [Internet]. 2010 [acesso em 2021 Mar 13]; 1(2):115-25. Disponível em: https://scielo.conicyt.cl/pdf/cienf/v16n2/ art_12.pdf

3 Costa DO, Souza FIS, Pedroso GC, Strufaldi MWL. Transtornos mentais na gravidez e condições do recém-nascido: estudo longitudinal com gestantes assistidas na atenção básica. Ciênc. saúde colet. [Internet]. 2018 [acesso em 2021 Mar 13]; 23(3): 691-700. Disponível em: https:// www.scielosp.org/article/csc/2018.v23n3/691-700/

4 Leite MG, Rodrigues DP, Sousa AAS, Melo LPT, Fialho AVM. Sentimentos advindos da maternidade: revelações de um grupo de gestantes. Psicol. Estud. [Internet]. 2014 [acesso em 2021 Mar 13]; 19(1):115-24. Disponível em: https://www.scielo.br/scielo.php?pid=S1413$-73722014000100013 \&$ script $=$ sci_abstract\&tlng=pt 
5 Schiavo RA, Rodrigues OMPR, Perosa GB. Variáveis associadas à ansiedade gestacional em primigestas e multigestas. Trends Psychol. [Internet]. 2018 [acesso em 2021 Mar 13]; 26(4):20912104. Disponível em: http://pepsic.bvsalud.org/scielo.php?script=sci_arttext\&pid=S1413-389X2018000400014\&lng=pt\&nrm=iso

6 Andrade RD, Santos JS, Maia MAC, Mello DF. Fatores relacionados à saúde da mulher no puerpério e repercussões na saúde da criança. Esc. Anna Nery [Intenet]. 2015 [acesso em 2021 Mar 13]; 19(1):181-6. Disponível em: https://www.scielo.br/pdf/ean/v19n1/1414-8145ean-19-01-0181.pdf

7 Almeida NMC, Arrais AR. O Pré-Natal Psicológico como Programa de Prevenção à Depressão Pós-Parto. Psicol. cienc. prof. [Internet]. 2016 [acesso em 2021 Mar 13]; 36(4):847- 63. Disponível em:< https://www.scielo.br/scielo.php?pid=S1414-98932016000400847\&script=sci_arttext>

8 Campos BC, Rodrigues OMPR. Depressão pós-parto materna: crenças, práticas de cuidado e estimulação de bebês no primeiro ano de vida. Psico Porto Alegre [Internet]. 2015 [acesso em 2021 Mar 13]; 46(4): 483-92. Disponível em: http://pepsic.bvsalud.org/scielo.php?script=sci_ar ttext\&pid=S0103-53712015000400009

9 Cantilino A, Zambaldi CF, Sougey EB, Junior JR. Transtornos psiquiátricos no pós-parto Rev. psiquiatr. clín.[Internet]. 2010 [acesso em 2021 Mar 13]; 37(6): 278-84. Disponível em: https:// www.scielo.br/scielo.php?script=sci_arttext\&pid=S0101-60832010000600006

10 Andrade LH, Viana MC, Silveira CM. Epidemiologia dos transtornos psiquiátricos na mulher. Arch. Clin. Psychiatry [Internet]. 2006 [acesso em 2021 Mar 13]; 33(2):43-54. Disponível em: https://www.scielo.br/pdf/rpc/v33n2/a03v33n2.pdf

11 Silva MA, Demitto M, Agnolo C, Torres M, Carvalho MDB, Pelloso SM. Tristeza materna em puérperas e fatores associados. Rev. port. enferm. saúde mental. [Internet]. 2017 [acesso em 2021 Mar 13]; 1(18): 08-13. Disponível em: http://www.scielo.mec.pt/scielo.php?script=sci_art text\&pid=S1647-21602017000300002

12 Serdán EM. Diferencias entre la depresión postparto, la psicosis postparto y la tristeza postparto. Perinatol. Reprod. Hum. [Internet]. 2013 [acesso em 2021 Mar 13];27(3):185-93. Disponível em: http://www.scielo.org.mx/scielo.php?script=sci_arttext\&pid=S0187-53372013000300008

13 Morais AODS, Simões VMF, Rodrigues LS, Batista RFL, Lamy ZC, Carvalho CA et al., Sintomas depressivos e de ansiedade maternos e prejuízos na relação mãe/filho em uma coorte pré-natal: uma abordagem com modelagem de equações estruturais. Cad. Saúde Pública [Internet]. 2017 [acesso em 2021 Mar 13]; 33(6) e00032016: 1-16. Disponível em: https://www.scielo.br/pdf/csp/ v33n6/1678-4464-csp-33-06-e00032016.pdf

14 Mendes KDS, Silveira RCCP, Galvão CM. Revisão integrativa: método de pesquisa para a incorporação de evidências na saúde e na enfermagem. Texto \& contexto enferm. [Internet]. 2008 [acesso em 2021 Mar 13]; 17(4):758-764. Disponível em: https://www.scielo.br/scielo. php?script=sci_arttext\&pid=S0104-07072008000400018

15 Zareba K, Banasiewicz J, Rozenek H, Wójtowicz S, Jakiel G. Peripartum Predictors of the Risk of Postpartum Depressive Disorder: Results of a Case-Control Study. Int $\mathrm{j}$ environ res public health [Internet]. 2020 [acesso em 2021 Mar 13]; 17(23):8726. Disponível em: https://pubmed. ncbi.nlm.nih.gov/33255386/

16 Pan WL, Chang CW, Chen SM, Gau ML. Assessing the effectiveness of mindfulness-based programs on mental health during pregnancy and early motherhood - a randomized control trial. BMC pregnancy childbirth [Internet]. 2019 [acesso em 2021 Mar 13]; 19(1):346. Disponível em: https://pesquisa.bvsalud.org/brasil/resource/pt/mdl-31601170 
17 Akbarzadeh M, Sima, KR, Moattari M, Zare N. Investigation of breastfeeding training based on BASNEF model on the intensity of postpartum blues. East Mediterr Health J [Internet]. 2018 [acesso em 2021 Mar 13]; 23(12):830-5. Disponível em: https://pesquisa.bvsalud.org/brasil/resource/pt/mdl-29528093

18 Riazanova O, Alexandrovich Y, Loscovich A. The relationship between labor pain management, cortisol level and risk of postpartum depression development: a prospective nonrandomized observational monocentric trial. Rom J Anaesth Intensive Care [Internet]. 2018 [acesso em 2021 Mar 13]; 25(2):123-30. Disponível em: https://pubmed.ncbi.nlm.nih.gov/30393769/

19 Dowlati Y, Ravindran AV, Segal ZV, Stewart DE, Steiner M, Meyer JH. Selective dietary supplementation in early postpartum is associated with high resilience against depressed mood. Proc Natl Acad Sci U S A [Internet]. 2017 [acesso em 2021 Mar 13]; 114(13):3509-14. Disponível em: https://www.pnas.org/content/114/13/3509

20 Fiala A, Svancara J, Klavova J, Kasparek T. Sociodemographic and delivery risk factors for developing postpartum depression in a sample of 3233 mothers from the Czech ELSPAC study. BMC Psychiatry [Internet]. 2017 [acesso em 2021 Mar 13]; 17(1):104. Disponível em: https://pesquisa. bvsalud.org/brasil/resource/pt/mdl-28327118

21 Mughal S, Azhar Y, Siddiqui W. Postpartum Depression. In: StatPearls.Stat Pearls Publishing, Treasure Island, Florida, 2020 [acesso em 2021 Mar 13]. Disponível em: https://bmcpsychiatry.biomedcentral.com/articles/10.1186/s12888-017-1261-y

22 Maliszewska K, Freund MS, Bidzan M, Preis K. Relationship, social support, and personality as psychosocial determinants of the risk for postpartum blues. Ginekol. pol. [Internet]. 2016 [acesso em 2021 Mar 13]; 87(6):442-7. Disponível em: https://journals.viamedica.pl/ginekologia_polska/article/view/47850

23 Kumar N, Nagaraj AKM, Koudike U, Majgi SM. Psychiatric Morbidity and Correlates in Postpartum Women in a Tertiary Care Hospital. Indian J Psychol Med. [Internet]. 2016 [acesso em 2021 Mar 13]; 38(4):309-14, 2016. Disponível em: https://pubmed.ncbi.nlm.nih.gov/27570341/

24 Masmoudi J, Charfeddine F, Trabelsi S, Feki I, Ayad BB, Guermazi M et al. Postpartum depression: prevalence and risk factors. A prospective Study concerning 302 Tunisian parturientes. Tunis Med.[Internet]. 2014 [acesso em 2021 Mar 13]; 92(10):615-21, 2014. Disponível em: https:// pubmed.ncbi.nlm.nih.gov/25860676/

\section{DADOS DOS AUTORES}

\section{Roberto Nascimento de Albuquerque}

Doutor em enfermagem. Professor titular do Centro Universitário de Brasília (CEUB), Brasília/DF-Brasil. roberto.albuquerque@ceub.edu.br

\section{Daiana Valéria da Silva Rollemberg}

Acadêmica de enfermagem do Centro Universitário de Brasília (CEUB), Brasília/DF-Brasil. daiana. rollemberg@sempreceub.com

Submetido em: 24-3-2021

Aceito em: 18-5-2021 